\title{
Location-specific immunodetection of cocaine on banknotes
}

\author{
Susan van der Heide ${ }^{a}$, Andrew Cunningham ${ }^{b}$, Sheila Hardwick ${ }^{c}$ and David A. Russella* \\ a School of Chemistry, University of East Anglia, Norwich Research Park, Norwich, Norfolk; \\ NR4 7TJ, UK. \\ ${ }^{b}$ European Monitoring Centre for Drugs and Drug Addiction (EMCDDA), Lisbon, Portugal. \\ ${ }^{c}$ Home Office Centre for Applied Science and Technology (CAST), Sandridge, St. Albans, \\ Herts, AL4 9HQ, UK.
}

\begin{abstract}
A novel in-gel bioanalytical immunodetection method has been developed to determine both the presence and the location of cocaine on the surface of banknotes. The cocaine was 'fixed' to the surface of the banknote via a coating of a polyacrylamide gel matrix. Immunostaining of the immobilised cocaine on the banknote surface was performed using an anti-cocaine primary antibody, either pre-labelled with horse radish peroxidase (HRP) or in conjunction with a HRP-labelled secondary antibody. Visualisation of the location of the cocaine was achieved through chemiluminescence imaging of the banknote following application of a chemiluminescent substrate. The novel method was applied to the detection of cocaine on partial and whole banknote samples obtained from general circulation. Newly minted banknotes, with or without spiked cocaine, were used as positive and negative controls, respectively. The results obtained, for the first time, demonstrate the successful location-specific immunostaining of cocaine on banknotes. A preliminary analysis of six UK banknotes, obtained from general circulation, suggests that cocaine can be present at variable locations across the whole of the banknote.
\end{abstract}

\section{Introduction}

The supply and consumption of cocaine is the cause of health, social and economic problems on a global scale. ${ }^{1-3}$ In order to combat the effect of usage and trade, many countries implement laws that allow the seizure of circumstantial evidence associated with cocaine trafficking. ${ }^{4,5}$ Cocaine, detected on paper currency, can be indicative of drug use or trade and is thus used by police to provide circumstantial evidence, in a court of law, that the money was involved in drug trafficking or trade. ${ }^{9,10}$ However, presenting cocaine on banknotes as evidence requires that the concentrations of cocaine on the confiscated banknotes to be distinguishable from that present on notes in general circulation. The development of analytical methods for the quantitative analysis of cocaine on banknotes has led to efforts being made towards the identification of different degrees of contamination. 
Analytical methods that enable the differentiation between 'drug money' (i.e. money which has been in direct contact with cocaine) and cocaine contamination from general circulation, and are in turn suitable for daily analysis in a forensic laboratory are crucial in providing such evidence. ${ }^{6-13}$ It is therefore important that a greater range of suitable methods for this type of analysis are developed.

At present, the analysis of cocaine on banknotes performed by law enforcement agencies is focussed on the use of ion-mobility spectrometry (IMS) as a means of quantification. ${ }^{14}$ The method involves taking a representative sample of cocaine along two strips on the side of the banknote by vacuum sampling. ${ }^{14,15}$ Recently, it has been suggested that the area sampled may not provide an adequate representative sample of the cocaine concentration present on the note in its entirety. ${ }^{15}$ For example, the primary location of cocaine on banknotes could be around the edges of the note rather than spread uniformly across it, in which case the cocaine present would be greater than indicated using the present method of sampling. An analytical method which enables the areas on banknotes where cocaine contamination most frequently occurs is therefore necessary to confirm the authenticity of the results obtained using the current means of analysis. It is essential that the analytical method is developed to enable both the presence and location of the cocaine on banknotes to be determined.

Biological methods of immunodetection such as immunohistochemistry (IHC) and immunocytochemistry (ICC) enable the location-specific detection of cellular antigens in specimens such as fixed tissue sections. Immunodetection of this nature enables the context of the antigenic material to be established in relation to other structural components in the sample. ${ }^{16-18}$ The specific and sensitive nature of immunological detection methods facilitated by the antigen recognition ability of antibodies makes them highly suited for the detection of molecules at low concentrations in complex sample matrices. ${ }^{19,20}$

The immunodetection of antigenic proteins in polyacrylamide gels is most commonly performed via indirect methods such as western blotting, where proteins within the gel are first transferred to a membrane prior to immunostaining. ${ }^{21}$ Certain limitations of western blotting have led to the development of a method for the in-gel immunodetection of antigenic material, first reported by Burridge following separation by SDS polyacrylamide gel electrophoresis. ${ }^{21-23}$ The technique involves fixation of the protein fragments within the gel via an incubation step in trichloroacetic acid or isopropanol with acetic acid. ${ }^{21,22}$ It is thought that the action of the fixative may also act to improve the permeability of the gel to antibodies. ${ }^{21,22}$ Immunodetection is then facilitated by the direct incubation of the gel 
containing the antigenic material in solutions of primary antibody and then HRP-labelled secondary antibody. ${ }^{22-25}$ Since its inception, in-gel immunodetection has been adapted to include different visualisation techniques such as radioactive and fluorescent labelling, and has been applied for the detection of several different cellular antigens. ${ }^{24,25}$ The application of IHC methods for the detection of antigen on fabric has been previously described by Miller et al., who reported the successful immunostaining of brain cells on cotton fabric using IHCbased methodology. ${ }^{26}$ The stability of cocaine in a proteinaceous tissue network formed by fixation using formaldehyde has also been studied by Viel et al. and Cingolani et al., who separately report the successful extraction of cocaine and benzoylecgonine from liver specimens up to seven days after fixation, indicating the suitability of a cross-linked hydrogel matrix for cocaine detection. ${ }^{27,28}$

Previously, we have shown that gold nanoparticles ${ }^{29,30}$ and magnetic particles ${ }^{31-33}$ functionalised with antibodies can be used to detect drugs and drug metabolites. These metallic-particle antibody conjugates were used to detect these analytes in the sweat deposited within a latent fingerprint. ${ }^{29,31-34}$ Through the use of fluorescently labelled secondary antibodies and fluorescence microscopy, not only was the presence of the drugs and drug metabolites determined but, simultaneously, the fingerprints could be imaged with high resolution. Banknotes are typically made of a blend or cotton and linen rag. ${ }^{10,35}$ Such materials may entrap antibody-particle conjugates. Additionally, banknotes are known to typically possess fluorescent anti-counterfeiting marks or images that may spectrally interfere with a fluorescence based imaging method used for the detection of cocaine. Consequently, we have chosen to develop a new method, based on chemiluminescence, for the detection of cocaine on the surface of banknotes.

In this present study the development of a novel immunostaining method for the locationspecific detection of cocaine on the surface of banknotes is described. The cocaine was fixed to the surface of the banknote via the application of a polyacrylamide gel matrix. Immunostaining of the immobilised cocaine on the banknote surface was subsequently performed using an anti-cocaine primary antibody, either pre-labelled with horse radish peroxidase (HRP) or in conjunction with a HRP-labelled secondary antibody. Visualisation was achieved by the application of a chemiluminescent substrate, producing a sensitive luminescent signal from the areas of the banknote where the cocaine was present. The developed method was applied to the detection of cocaine firstly on partial banknote samples spiked with cocaine standard. Whole banknote samples, from general circulation, were then analysed alongside whole banknote samples on which half of the note had been spiked with a sample of 'street' cocaine. Newly minted banknotes, with or without cocaine, 
were used as positive and negative controls, respectively. The results obtained in this preliminary study, for the first time, demonstrate the successful location-specific immunodetection of cocaine on banknotes.

\section{Experimental}

\section{Materials}

All reagents were of analytical grade, purchased from Sigma-Aldrich (UK) and used as received unless otherwise stated. Analytical grade deionised water $\left(\mathrm{dH}_{2} \mathrm{O}\right)$, methanol $(\mathrm{MeOH})$, hydrochloric acid $32 \%(\mathrm{HCl}(\mathrm{aq}))$, sodium dihydrogen orthophosphate dihydrate $\left(\mathrm{NaH}_{2} \mathrm{PO}_{4} \cdot 2 \mathrm{H}_{2} \mathrm{O}\right)$, di-sodium hydrogen orthophosphate anhydrous $\left(\mathrm{Na}_{2} \mathrm{HPO}_{4}\right)$, sodium hydrogen carbonate $\left(\mathrm{NaHCO}_{3}\right)$, sodium chloride $(\mathrm{NaCl})$ and glycerol were all purchased from Fisher Scientific (UK). Sodium dodecyl sulfate (SDS) was purchased from Pharmacia Biotech (UK). Rabbit polyclonal anti-cocaine antibody was purchased in $1 \mathrm{~mL}$ units of serum from Europa Bioproducts (UK). Sheep polyclonal anti-cocaine antibody and horseradish peroxidase (HRP)-labelled mouse monoclonal anti-sheep lgG were purchased from Abcam (UK) and Jackson ImmunoResearch laboratories (USA), respectively. 'Slide-A-Lyzer' mini dialysis units (10,000 MWCO), Coomassie brilliant blue R250, bromophenol blue sodium salt, ammonium persulfate (APS) and horseradish peroxidase (HRP) were all purchased from Thermo Scientific (UK). Novex Sharp unstained protein standard (molecular weight marker) and Novex enhanced chemiluminescence (ECL) substrate reagent were purchased from Invitrogen (UK). $18 \times 16 \mathrm{~cm}$ glass plates and $16 \mathrm{~cm} \times 8 \mathrm{~cm} \times 1.5 \mathrm{~mm}$ gel spacers were purchased from Web Scientific (UK). N,N,N'-tetramethylethylenediamine (TEMED) was purchased from Bio Rad (UK). Cerilliant Cocaine standard (1 $\mathrm{mg} \mathrm{mL}^{-1}$ in acetonitrile) was purchased from LGC Standards (UK).

Newly minted £20 GBP banknotes, together with GBP banknotes of varying denominations from general circulation were kindly provided by the Bank of England (Notes Division, Banking Services; Loughton, UK). Approximately $1 \mathrm{~g}$ of seized 'street' cocaine (of unknown purity) was kindly provided by the Norfolk Police Constabulary (Serious and Organised Crime Directorate, Wymondham, Norfolk, UK).

Native-PAGE gels were cast in a Hoefer S245 dual gel cassette and performed using a Hoefer Mighty Small II vertical electrophoresis system. $16 \times 18 \mathrm{~cm}^{2}$ native-PAGE gels were cast using a Hoefer SE600 dual cassette. Gel electrophoresis was performed using an Amersham Pharmacia Biotech EPS 601 power supply. Colorimetric imaging of the native- 
PAGE gels was performed using an HP photosmart C4580 scanner. Chemiluminescent imaging was performed using a Fuji LAS-3000 imaging system; images were obtained at $30 \mathrm{~s}$ intervals in chemiluminescent increment mode and at high sensitivity for a total exposure time of $5 \mathrm{~min}$. All images were contrast adjusted by +2 . UV-Vis absorption spectra were recorded on a Hitachi U-3000 spectrophotometer at room temperature. Quartz cuvettes with a $10 \mathrm{~mm}$ path length were used. HRP-labelled anti-cocaine antibody was separated from unconjugated HRP by gel filtration using a GE Health Life Sciences Superdex 200 column (350 mL volume) and an Amersham Pharmacia Biotech AKTA Explorer. $20 \mathrm{mM}$ phosphate buffered saline with $150 \mathrm{mM} \mathrm{NaCl}(\mathrm{pH}$ 7.2) was used as a running buffer. Absorbance intensity of the fractions was measured at $280 \mathrm{~nm}$.

\section{Development of the in-gel immunodetection method using native-PAGE gel electrophoresis}

Control samples containing primary anti-cocaine antibody or secondary HRP-labelled antibody were imbedded into a polyacrylamide gel using a modified version of the nativepolyacrylamide gel electrophoresis (native-PAGE) method described by Walker. ${ }^{36}$ The gels were cast as $8 \times 10 \mathrm{~cm}^{2}$ homogenous $7.5 \% \mathrm{w} / \mathrm{v}$ acrylamide Tris- $\mathrm{HCl}$ slab-gels using $0.75 \mathrm{~mm}$ spacers. The pre-prepared gel mix was added slowly to the cassette and allowed to set at room temperature. $10 \mu \mathrm{L}$ of the molecular weight marker was loaded into the first well of the gel. $15 \mu \mathrm{L}$ of sample, diluted 1:1 in 1x native-PAGE Laemmli sample buffer ${ }^{37}$ was then added to each remaining well. Electrophoresis was performed at $120 \mathrm{~V}$ constant voltage for approximately $3 \mathrm{~h}$. Gels which were not required for in-gel immunodetection were stained using Coomassie brilliant blue R250 protein stain.

In-gel immunodetection was performed on unstained gels using a modified version of the method described by Desai et al.. ${ }^{21}$ Following electrophoresis, the gel was removed from the cassette and transferred to a gel box. The gel was then submerged in approximately $50 \mathrm{~mL}$ of $50 \%$ isopropanol/water for $15 \mathrm{~min}$ at room temperature. The isopropanol/water incubation was followed by a $15 \mathrm{~min}$ wash step in approximately $50 \mathrm{~mL}$ of $\mathrm{dH}_{2} \mathrm{O}$. The gel was then incubated for $2 \mathrm{~h}$ at room temperature in a $20 \mathrm{~mL}$ solution of rabbit polyclonal anti-cocaine primary antibody $\left(1 \mu \mathrm{g} \mathrm{mL} \mathrm{m}^{-1}\right.$ in sample buffer, $0.5 \% \mathrm{w} / \mathrm{v} \mathrm{BSA} / 1 \mathrm{x}$ Tris buffered saline (TBS)/0.05\% v/v Tween-20). Following incubation with the primary antibody, the gel was washed three times for $10 \mathrm{~min}$ in approximately $50 \mathrm{~mL}$ of wash buffer (TBS/0.05 v/v Tween20). $20 \mathrm{~mL}$ of diluted HRP-labelled anti-rabbit lgG secondary antibody (1:100 in sample buffer) was then added to the gel and incubated for $1 \mathrm{~h}$ at RT. The gel was then washed a further three times as previously described. 
An enhanced chemiluminescence (ECL) substrate solution was applied for the detection of $\mathrm{HRP}$ within the gels. For this, $10 \mathrm{~mL}$ of the ECL substrate solution was prepared by mixing $5 \mathrm{~mL}$ of reagent $A$ (stable peroxidase buffer) with $5 \mathrm{~mL}$ of reagent $B$ (luminol enhancer). This was added to the gel immediately after the final wash step, and was followed by a $5 \mathrm{~min}$ incubation in the dark at room temperature. The ECL solute ion was removed after incubation, and the gel was briefly rinsed (for around $15 \mathrm{~s}$ ) in $\mathrm{dH}_{2} \mathrm{O}$ to remove any excess of the ECL solution. The gel was then placed between acetate sheets and imaged.

\section{HRP labelling of polyclonal anti-cocaine antibody}

The direct labelling of the primary anti-cocaine antibody with HRP to form an antibody-HRP conjugate was performed to simplify the immunodetection procedure. Anti-cocaine lgG was labelled with HRP using the periodate-mediated method of conjugation as reported by Tijssen and Kurstak, ${ }^{38}$ and Wisdom. ${ }^{39} 2 \mathrm{mg}$ of HRP was dissolved in $500 \mu \mathrm{L}$ of sterile-filtered $\mathrm{dH}_{2} \mathrm{O} .100 \mu \mathrm{L}$ of freshly prepared $0.1 \mathrm{M}$ sodium periodate was added to the HRP solution and stirred for $20 \mathrm{~min}$ at room temperature, during which a colour change of orange to green was observed. The resulting solution was dialysed in mini-dialysis units overnight at $4{ }^{\circ} \mathrm{C}$ against $2 \mathrm{~L}$ of $1 \mathrm{mM}$ sodium acetate buffer ( $\mathrm{pH} 4.4) .50 \mu \mathrm{L}$ of the anti-cocaine antibody was made up to $500 \mu \mathrm{L}$ in $10 \mathrm{mM}$ sodium carbonate buffer $(\mathrm{pH}$ 9.5). The $\mathrm{pH}$ of the dialysed enzyme was adjusted to $9.0-9.5$ by adding $10 \mu \mathrm{L}$ of $0.2 \mathrm{M}$ sodium carbonate buffer (pH 9.5), after which the solution containing the antibody was immediately added. The mixture was then stirred for $2 \mathrm{~h}$ at room temperature. $50 \mu \mathrm{L}$ of freshly prepared sodium borohydride solution ( $4 \mathrm{mg} \mathrm{mL}^{-1}$ in $\mathrm{dH}_{2} \mathrm{O}$ ) was added and the mixture was stirred for a further $2 \mathrm{~h}$ at room temperature. After conjugation, the resulting HRP-labelled antibody was separated from unbound HRP by gel filtration. The presence of protein in the eluted fractions from the column was monitored by absorbance at $280 \mathrm{~nm}$. The fractions from the first peak at $A_{280}$ were selected, and the presence of the HRP-labelled antibody was confirmed by SDS-PAGE and by UV-Vis spectrophotometry at $A_{280}$ and $A_{403}$. Fractions found to be containing the hapten were then concentrated to a $1 \mathrm{~mL}$ volume by centrifugation in a Vivaspin20 tube at 2,500 $\mathrm{xg}$ for $40 \mathrm{~min}$ at $4{ }^{\circ} \mathrm{C}$. The $\mathrm{Rz}$ value, which is a measure of haem content (and an indicator of the purity of the preparation), was determined using the ratio of the absorbance intensity at $A_{275}: A_{403} .{ }^{19}$ The concentration of the labelled antibody was estimated based on the absorption of HRP at $403 \mathrm{~nm}$ using the extinction coefficient $102 \mathrm{mM}^{-1} \mathrm{~cm}^{-1} .{ }^{40}$ A typical satisfactory $\mathrm{Rz}$ value of 0.3 and a concentration of $2 \mu \mathrm{M}$ of labelled hapten was obtained. $5 \mathrm{mg}$ of BSA was added to the solution of labelled antibody to make a final BSA concentration of $5 \mathrm{mg} \mathrm{mL}^{-1}$. The solution was divided into $1 \mathrm{~mL}$ aliquots and stored at $-20^{\circ} \mathrm{C}$. 


\section{Preparation of spiked partial banknote and whole banknote samples}

In the UK, the Bank of England are the only authority who can destroy Bank of England banknotes. For the work reported herein, the Bank of England kindly provided the banknotes, both newly minted and those that had been used in general circulation. Following completion of the research, all partial and whole banknotes were returned to the Bank of England to be removed from circulation and destroyed. Whole or partial banknote samples were spiked with 'street' cocaine using the approach described by Ebejer et al.. ${ }^{13}$ For the partial banknote samples, £5 GBP notes were first cut width-wise into ca. $4 \mathrm{~cm}$ wide strips before spiking. Newly minted £20 GBP notes were used as whole banknote samples. A flat glass plate was dusted with $25 \mathrm{mg}$ of 'street' cocaine to represent a 'contaminated surface.' The partial or whole banknote was then rubbed over the surface at random, in order to achieve sporadic but overall coverage. The same process was performed for both sides of the note. The contaminated glass plate was weighed before and after spiking to enable the quantity of cocaine adhered to the note to be estimated.

\section{In-gel immunodetection of cocaine on partial banknote and whole banknote samples}

In-gel immunodetection of cocaine on banknote samples was performed using a method based on that developed for the native-PAGE gels (as described above). Figure 1 shows a schematic overview of the developed method for the immunodetection on whole banknotes. An appropriately sized gel cassette $\left(8 \times 10 \mathrm{~cm}^{2}\right.$ for partial banknote samples, and $16 \times 18 \mathrm{~cm}^{2}$ for whole banknote samples) was assembled using $1.5 \mathrm{~mm}$ spacers. A $7.5 \%$ acrylamide native-PAGE gel mix was then prepared and poured slowly into the cassette. The pre-spiked partial banknote sample or whole banknote sample was then lowered slowly into the solution and pushed down with tweezers until completely immersed. Air bubbles in the gel mix were removed by gently tapping the cassette, or by piercing the bubbles using a narrow pipette tip. A thin layer of ethanol (ca. $3 \mathrm{~mm}$ ) was floated on top of the gel before the gel was left to set for ca. $40 \mathrm{~min}$ at room temperature.

Once set, the gel containing the sample was gently removed from the cassette and transferred to a gel box. For the whole banknote samples, excess gel (beyond ca. $1.5 \mathrm{~cm}$ of the edge of the banknote) was removed using a scalpel prior to transfer to the gel box. Once transferred, the gel was then submerged in $50 \%$ isopropanol in $\mathrm{dH}_{2} \mathrm{O}(50 \mathrm{~mL}$ for partial banknote samples, or $100 \mathrm{~mL}$ for whole banknote samples) for $15 \mathrm{~min}$ at room temperature. This was followed by a 15 min wash step in $\mathrm{dH}_{2} \mathrm{O}$. The gel was then incubated for $2 \mathrm{~h}$ at room temperature in either $20 \mathrm{~mL}$ for partial banknote samples, or $40 \mathrm{~mL}$ for whole banknote samples of HRP-labelled sheep anti-cocaine antibody diluted to 1:200 in sample buffer. After 
incubation in the antibody solution, the gel was then washed three times for 10 min in wash buffer (50 mL for partial banknote samples, or $100 \mathrm{~mL}$ for whole banknote samples). The ECL substrate solution ( $10 \mathrm{~mL}$ for partial banknote samples, or $20 \mathrm{~mL}$ for whole banknote samples) was prepared by using a 1:1 dilution of stable peroxidase buffer (reagent $A$ ) with luminol enhancer (reagent B). The ECL solution was added to the gel and incubated for $5 \mathrm{~min}$ at room temperature in the absence of light. The solution was then removed and the gel was briefly rinsed (around $15 \mathrm{~s}$ ) in $\mathrm{dH}_{2} \mathrm{O}$, before being placed between acetate sheets and imaged at $30 \mathrm{~s}$ intervals for a total exposure time of $5 \mathrm{~min}$.

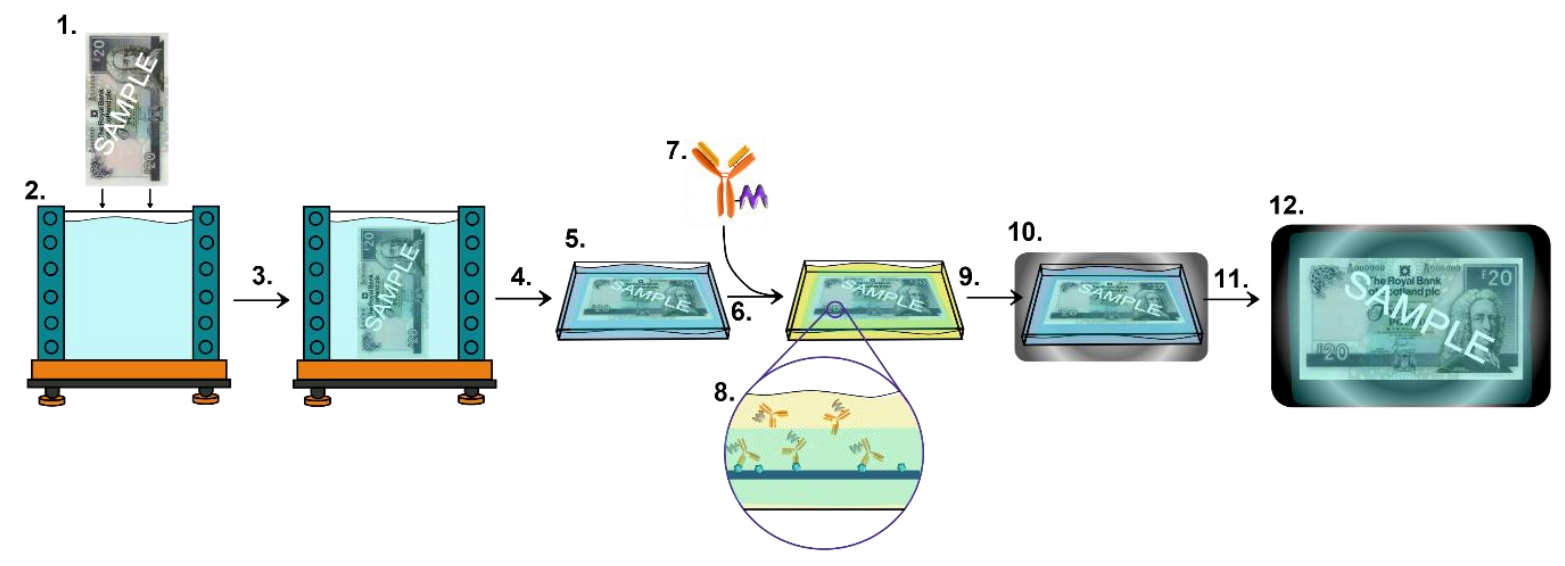

Figure 1. A schematic representation of the in-gel immunodetection method for the detection of cocaine on banknotes. The numbers indicate as follows; 1 . The banknote is submerged in a polyacrylamide gel solution; 2 . A $16 \times 18 \mathrm{~cm}^{2}$ cassette containing the polyacrylamide gel solution; 3 . The gel is set at room temperature; 4 . The gel, with the encased banknote, is removed from the cassette; 5 . The gel is placed in $50 \%$ isopropanol in $\mathrm{dH}_{2} \mathrm{O} ; 6$. Incubation followed by washing; 7 . The HRP-labelled anticocaine antibody is added; 8 . The antibody passes through the gel and binds to the cocaine on the banknote; 9 . Washing; 10 . The ECL substrate is added; 11. An incubation step (in the dark) and washing; 12. Chemiluminescent imaging. Figure adapted from van der Heide and Russell. ${ }^{41}$ 


\section{Results and discussion}

\section{Method development}

Polyacrylamide gels, commonly used for the separation of biomolecules by gel electrophoresis, were applied in this study as a matrix for 'fixing' the cocaine present on the surface of the banknotes. As the mechanical strength of covalently cross-linked acrylamide gels is known, the permeability of antibodies through the gels following treatment with $50 \%$ isopropanol in water was first investigated. The immunostaining of acrylamide gels was initially performed using the method of Desai et al. following separation by native-PAGE. ${ }^{21}$ The gels were run with a sample of rabbit polyclonal anti-cocaine primary antibody applied as the antigenic target, and a corresponding HRP-labelled anti-rabbit IgG secondary antibody as a means of immunolabelling. Solutions of BSA and of the HRP-labelled secondary antibody were included as negative and positive control samples, respectively. Immunostaining was performed using an enhanced chemiluminescent (ECL) substrate. The resulting gel image shows a negative version of the stained gel, in which the luminescent signal produced by the presence of HRP is converted into 'staining' of a grey or black appearance on a white background. A gel containing the equivalent set of standards was also separated by native-PAGE but stained for all protein using Coomassie brilliant blue R250 rather than immunostaining. Figure 2 shows a typical set of results for this analysis. The gel shown in Figure 2A was stained for protein using Coomassie brilliant blue R250 and run using the same set of samples as used for the immunostained gel shown in Figure $2 \mathrm{~B}$. In Figure $2 \mathrm{~A}$, the lanes contain as follows; BSA in lane 1, anti-cocaine primary antibody in lane 2 and HRP-labelled secondary antibody in lane 3. Protein staining, as indicated by the blue bands, was present in all three lanes of the gel. The staining obtained from both the primary antibody and the secondary antibody in lanes 2 and 3, respectively, was localised further up the gel than the BSA in lane 1 due to the higher molecular weight of the antibodies (Figure $2 \mathrm{~A})$. In the corresponding immunostained gel shown in Figure $2 \mathrm{~B}$, as expected, no staining was observed in lane 1 which contained the BSA as a negative control. Staining, indicative of the presence of HRP (as indicated by areas of dark grey or white coloration within the gel), was observed in lanes 2 and 3 of Figure 2B in which the primary and secondary antibody were present, respectively (Figure $2 \mathrm{~B}$ ). The staining observed in lane 3 of Figure 2B was expected to occur due to the presence of the HRP-labelled secondary antibody positive control within the gel, and indicated that the detection of HRP using the enzyme substrate had been successful. The location of the HRP in lane 3 of Figure 2B corresponded to the position of the total protein stain in lane 3 of Figure $2 \mathrm{~A}$, thereby confirming that the 
detection of HRP was indicative of the presence of the secondary antibody. The presence of HRP was also observed in lane 2 of the immunostained gel shown in Figure 2B, in which the primary anti-cocaine antibody was present. The presence of enzyme staining in this lane indicated that the primary antibody present in the gel had successfully been detected by the HRP-labelled secondary antibody via the in-gel method of immunodetection (Figure 2B). The location of the enzyme staining in lane 2 of Figure $2 \mathrm{~B}$ corresponded to the position of the total protein stain in lane 2 of Figure $2 \mathrm{~A}$, thereby confirming the successful detection of the primary antibody. Based on the results shown in Figure 2, it was thought that the applied method could successfully be used for the antibody-based detection of antigenic material within a polyacrylamide gel matrix.

A

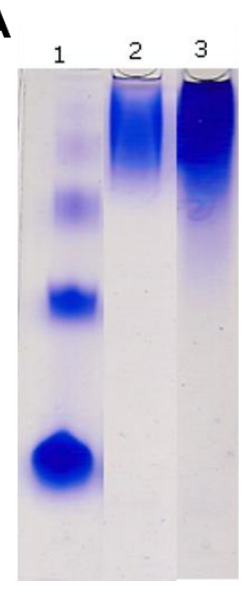

B

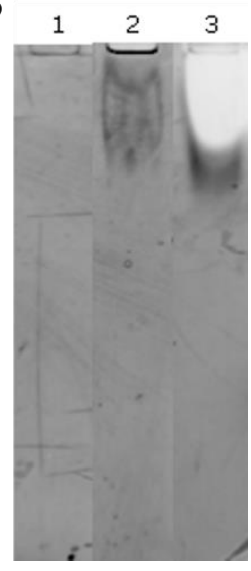

Figure 2. The in-gel immunodetection of cocaine-BSA in a $0.75 \mathrm{~mm}$ thick, $7.5 \% \mathrm{w} / \mathrm{v}$ acrylamide PAGE gel stained with an ECL enzyme substrate. The total protein in the gel, stained by Coomassie brilliant blue R250, is shown in (A). Immunostaining was performed on a gel identical to the one shown in $(A)$, the result of which is shown in (B). An exposure time of 3.5 min was used for the gel. The samples in the respective gels are labelled as follows; (1) BSA, (2) anti-cocaine primary antibody and (3) HRPlabelled secondary antibody. All samples were pre-diluted to $1 \mathrm{mg} \mathrm{mL}^{-1}$ in $\mathrm{PB}$ prior to analysis.

\section{Application of the in-gel immunodetection method for the analysis of partial banknote samples}

The immunodetection of cocaine, applied as a standard solution in acetonitrile onto partial banknote samples, initially proved unsuccessful using the HRP-labelled anti-cocaine 
antibody. The adherence of cocaine to banknotes has been described in previous studies to occur through the entrapment of crystals of the drug within the cotton and linen fibres comprising the banknote material. ${ }^{42,10}$ It was thought that cocaine applied as a solution would not adhere to the banknote in the same manner as the crystalline powder form of the drug, and would therefore yield a negative result. The procedure was thus repeated using a different portion of the same banknote sample spiked with crystalline 'street' cocaine rather than cocaine standard in solution. The street cocaine, which was received as a crystalline powder, was considered a more realistic representation of the form and purity of cocaine likely to be present on a banknote, rather than pure cocaine standard applied as a solution in solvent. Spiking was performed by rubbing both sides of a specific area of the note onto a surface covered in crystalline cocaine. It was noted that spiking in this way proved difficult and inaccurate, as the powder was prone to adhere to other parts of the banknote sample due to the small size of the area chosen for spiking. Due to the difficulty of accurately applying the crystalline cocaine to only one small area of a partial banknote, two separate partial banknotes, one with and one without cocaine, were instead analysed. This enabled the presence of cocaine to be determined in the absence of possible artefacts introduced by the method of spiking. A $£ 5$ note obtained from general circulation was cut into quarters, two of which were used for the analysis. Cocaine was then applied to both sides of the spiked 'positive' partial banknote.

The results of the analysis are shown in Figure 3, with the non-spiked banknote shown in Figure $3 \mathrm{~A}$ and $\mathrm{B}$ and the cocaine spiked 'positive' banknote in Figure $3 \mathrm{C}$ and D. The location of cocaine is indicated by the areas of dark grey staining, which results from the presence of the HRP-labelled antibody used to detect the cocaine within the gel which surrounds each banknote. The non-spiked and the 'positive' samples can be readily distinguished from one another based on the intensity of this dark grey staining on each of the notes. Overall, the staining on the 'positive' banknote (shown in Figure $3 \mathrm{C}$ and $\mathrm{D}$ ) is the darkest on the note itself rather than the surrounding gel, indicating that the spiked cocaine has successfully adhered to the banknote during spiking rather than becoming removed from the note during the gelation process. In addition, staining of a darker grey coloration was observed around the edge of the 'positive' banknote side shown in Figure 3C, and to a lesser degree on the edge of the other side of the same banknote shown in Figure 3D. This increase in the intensity of the staining around the edge of the 'positive' banknote suggests that this is an area of the note where the cocaine had predominantly adhered to during spiking. Alternatively, it is possible that the location of this staining could have also resulted from the migration of cocaine towards the edges of the banknote during gelation. Light grey staining, indicative of a low concentration of cocaine, was also observed on the non-spiked banknote 
shown in Figure $3 A$ and $B$; this staining may have resulted from cocaine contamination on the banknote, obtained from general circulation, which was detected during the analysis.
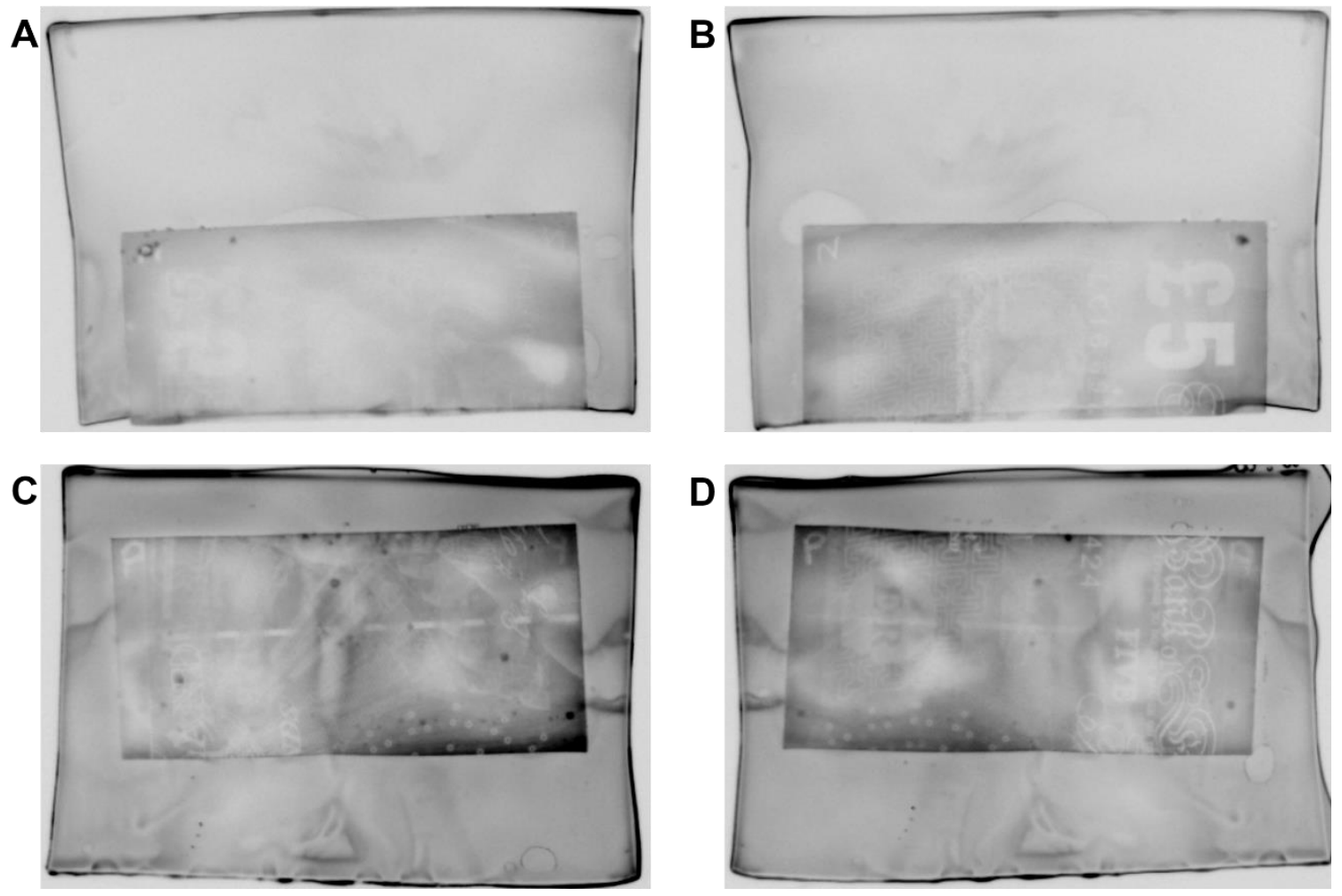

Figure 3. The immunostaining of partial banknote samples within $1.5 \mathrm{~mm}$ thick, $7.5 \% \mathrm{w} / \mathrm{v}$ polyacrylamide gels. (A) and (B) show each side of part of a banknote obtained from general circulation. (C) and (D) show each side of a different part of the same banknote spiked with $5 \mathrm{mg}$ of 'street' cocaine. Chemiluminescent imaging was performed using a Fuji LAS-3000 imaging system, on high sensitivity mode for a total exposure time of $5 \mathrm{~min}$.

\section{Application of the in-gel immunodetection method for the analysis of whole banknote samples}

Following the successful immunodetection of cocaine on partial banknote samples, the method was then adapted and applied to whole banknote samples. As banknotes from general circulation are known to contain background levels of cocaine contamination, ${ }^{10}$ newly minted notes (obtained directly from the Bank of England) were instead used as a truly negative sample matrix for the initial testing of the method. A clear difference in the intensity of staining was observed between newly minted negative control notes and those which had been spiked with 'street' cocaine over the whole of the note. These initial results suggested that the cocaine was successfully detected using the developed method. In order 
to confirm whether the staining was occurring at the location where cocaine was applied to the note, the analysis was repeated using newly minted banknotes where half of the note had been spiked with street cocaine and the other half left blank as a negative control area. The results, a typical example of which is shown in Figure $4(A-D)$, showed an area of intense staining at the edge of the spiked half of the note rather than over its entirety. The division between the positive cocaine-spiked side of the note (labelled 'P') and the nonspiked negative side of the note (labelled ' $N$ ') is indicated by the white line in Figure 4C and D. The area where the darkest staining occurred was at the edge of the banknote, rather than over the whole of the positive side of the note. This result suggested that the cocaine had migrated towards the edge of the note during gelation instead of remaining in its original spiked location.

The staining present on the area of the note shown in Figure 4C and D, which had been spiked with cocaine, was darker and more intense than that observed on the negative control note (shown in Figure 4A and B) although not the same degree of difference as observed in the partial banknote samples shown in Figure 3 . The greater intensity of the staining observed in Figure 3 was thought to relate to the level of wear of the banknotes, as the partial banknotes had been obtained from general circulation and were therefore more aged and worn than the newly minted notes. The aging of banknotes in general circulation has been reported to lead to a loosening and spreading of the tight weave of fibres that form the material of the note..$^{10,42}$ These effects of aging in turn lead to a greater porosity within the weave, in which cocaine crystals can more readily become entrapped and retained by the loosened fibres. 
A

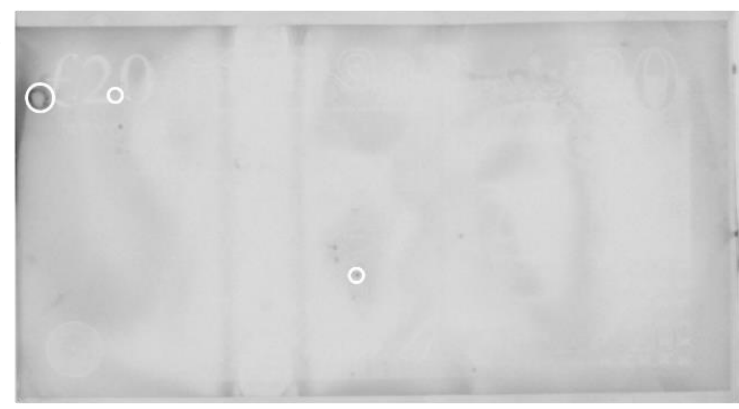

C I

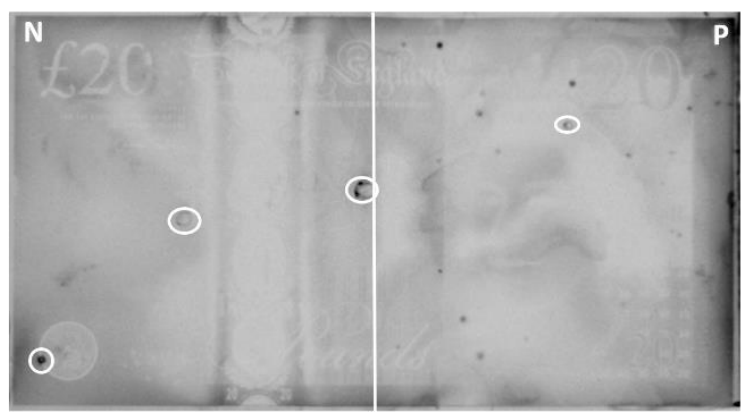

B
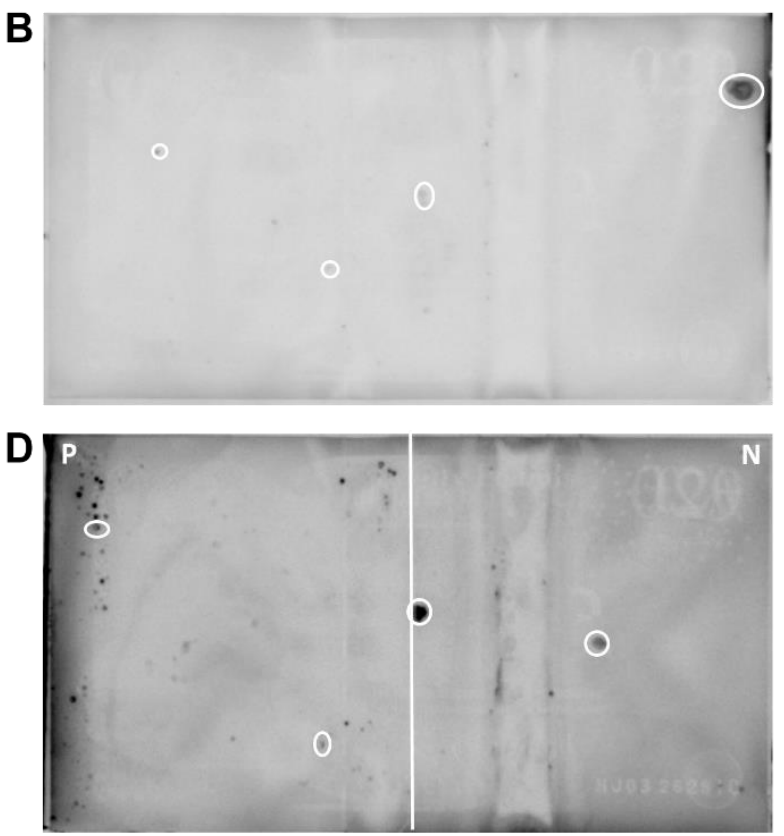

Figure 4. The immunostaining of newly minted $£ 20$ banknotes within $7.5 \% \mathrm{w} / \mathrm{v}$ polyacrylamide gels. (A) and (B) show each side of part of one banknote. (C) and (D) show each side of a second banknote, where half of the note is spiked with $10 \mathrm{mg}$ of street cocaine. The side of the note spiked with cocaine is labelled ' $\mathrm{P}$ ', and the side without cocaine is labelled ' $N$ '. Chemiluminescent imaging was performed using a Fuji LAS-3000 imaging system, on high sensitivity mode for a total exposure time of $5 \mathrm{~min}$. The white circles indicate where air bubbles are present in the gels.

The entrapment and adherence of cocaine crystals within the fibres of banknotes was suggested by Moss and by Sleeman et al. as relating to the age and therefore the structural integrity of the weave of the banknote. ${ }^{42,10}$ The immunodetection method was therefore applied to the analysis of six aged banknotes obtained from general circulation in order to confirm whether the use and aging of the notes affected the results obtained. This component of the present investigation enabled the analysis of samples with unknown levels of cocaine contamination to be performed. All of the notes analysed were of a worn and yellowed appearance and were more pliable as compared to the newly minted notes used in the previous experiments. The results of the immunostaining of two of the notes obtained from general circulation are shown in Figure 5. The two sides of the first note are shown in Figure $5 \mathrm{~A}$ and $\mathrm{B}$, while the two sides of the second banknote are shown in Figure $5 \mathrm{C}$ and $\mathrm{D}$. The nature of the staining observed on both the first (Figure $5 \mathrm{~A}$ and $\mathrm{B}$ ) and the second (Figure $5 \mathrm{C}$ and $\mathrm{D}$ ) banknotes from general circulation differed from that of the newly minted 
notes shown in Figure 4. For instance, small circular dots of intense dark grey staining were present on the older notes in Figure 5, as opposed to the overall grey hue seen on the newly minted notes. These localised spots of intense staining were thought to be the result of the detection of the levels of cocaine contamination present on the banknotes. The areas where the darkest staining occurred were on the right and left hand edge of the sides of the banknote shown in Figure $5 \mathrm{C}$ and $\mathrm{D}$, respectively. It is possible that this staining may have resulted from the migration of cocaine towards that edge of the banknote during gelation. However, due to the unknown nature of the sample and therefore the undetermined levels of cocaine contamination and location, the staining could be indicative of the presence of a large quantity of cocaine on that edge of the banknote. Staining of this nature was not observed in the other five banknotes that were analysed, suggesting that the images shown in Figure $5 \mathrm{C}$ and $\mathrm{D}$ highlight genuine detection of cocaine along the edge of this particular banknote. To confirm whether this pattern of staining due to the detection of cocaine was typical of banknotes from general circulation, further analyses of a larger number of banknotes would be required. The difference between the type of staining between the newly minted and the older banknotes from general circulation was thought to be due to the loosening of the banknote fibres that allows the entrapped cocaine crystals to remain in place even during application and subsequent gelation of the acrylamide gel. Due to the unknown levels of cocaine present on the banknotes obtained from general circulation, the genuine immunostaining of cocaine could not be confirmed based on these results alone. 

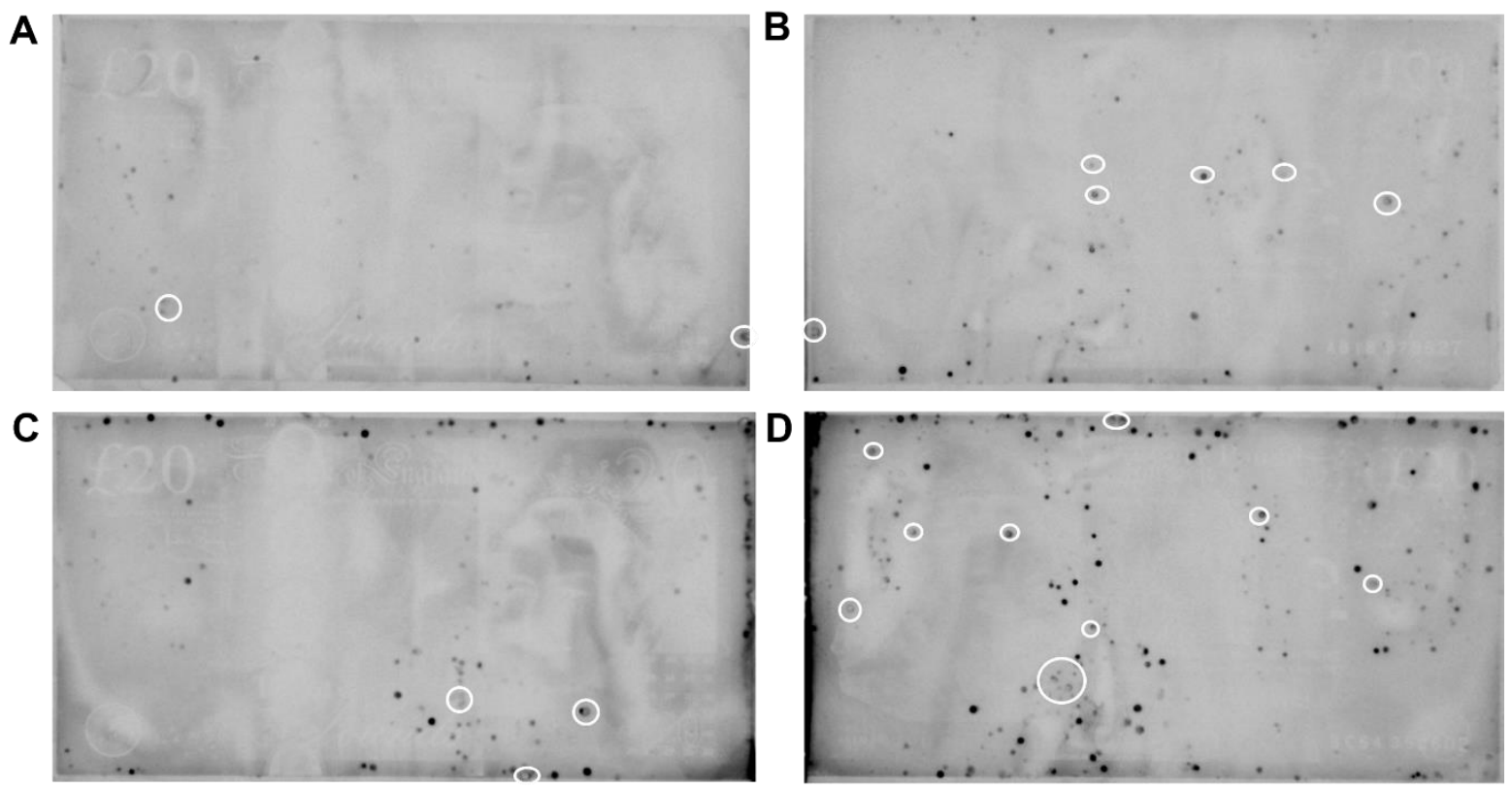

Figure 5. The immunostaining of two worn $£ 20$ banknotes obtained from general circulation within $7.5 \% \mathrm{w} / \mathrm{v}$ polyacrylamide gels. The two sides of the first banknote are shown $(A)$ and $(B)$, and each side of the second banknote in (C) and (D). Chemiluminescent imaging was performed using a Fuji LAS-3000 imaging system, on high sensitivity mode for a total exposure time of $5 \mathrm{~min}$. The white circles indicate where air bubbles are present in the gels.

In order to confirm whether the small, intense spots of immunostaining observed on the banknotes obtained from general circulation were the result of genuine immunodetection of cocaine on the surface of the note, the procedure was repeated on a set of half-spiked banknotes similar to the analysis previously performed on the newly minted notes (Figure 4). A typical result is shown in Figure 6 together with a non-spiked banknote from general circulation. The two sides of the non-spiked note are shown in Figure $6 \mathrm{~A}$ and $\mathrm{B}$, while the two sides of the half-spiked banknote are shown in Figure 6C and D. Overall, a greater number of stained areas were observed on the half-spiked banknote in Figure $6 \mathrm{C}$ and $\mathrm{D}$ as compared to the non-spiked note in Figure 6A and B. Further, a greater number of stained areas were also present on the cocaine-spiked half of the positive banknote (labelled ' $P$ ') than the non-spiked half of the banknote (labelled ' $N$ '). In particular, the side of the banknote shown in Figure 6D featured a greater number of dark spots of staining on the positively spiked half. The nature of the staining differed between the two banknotes; for example, the majority of the staining present in Figure $6 \mathrm{C}$ and $\mathrm{D}$ appeared as circular spots, whereas a 
high proportion of the staining in Figure $6 \mathrm{~A}$ and $\mathrm{B}$ was present as a band of darker colouration toward the bottom edge of the banknote. These results suggest that the locationspecific visualisation of cocaine on the surface of banknotes can be achieved using the developed in-gel immunodetection method.
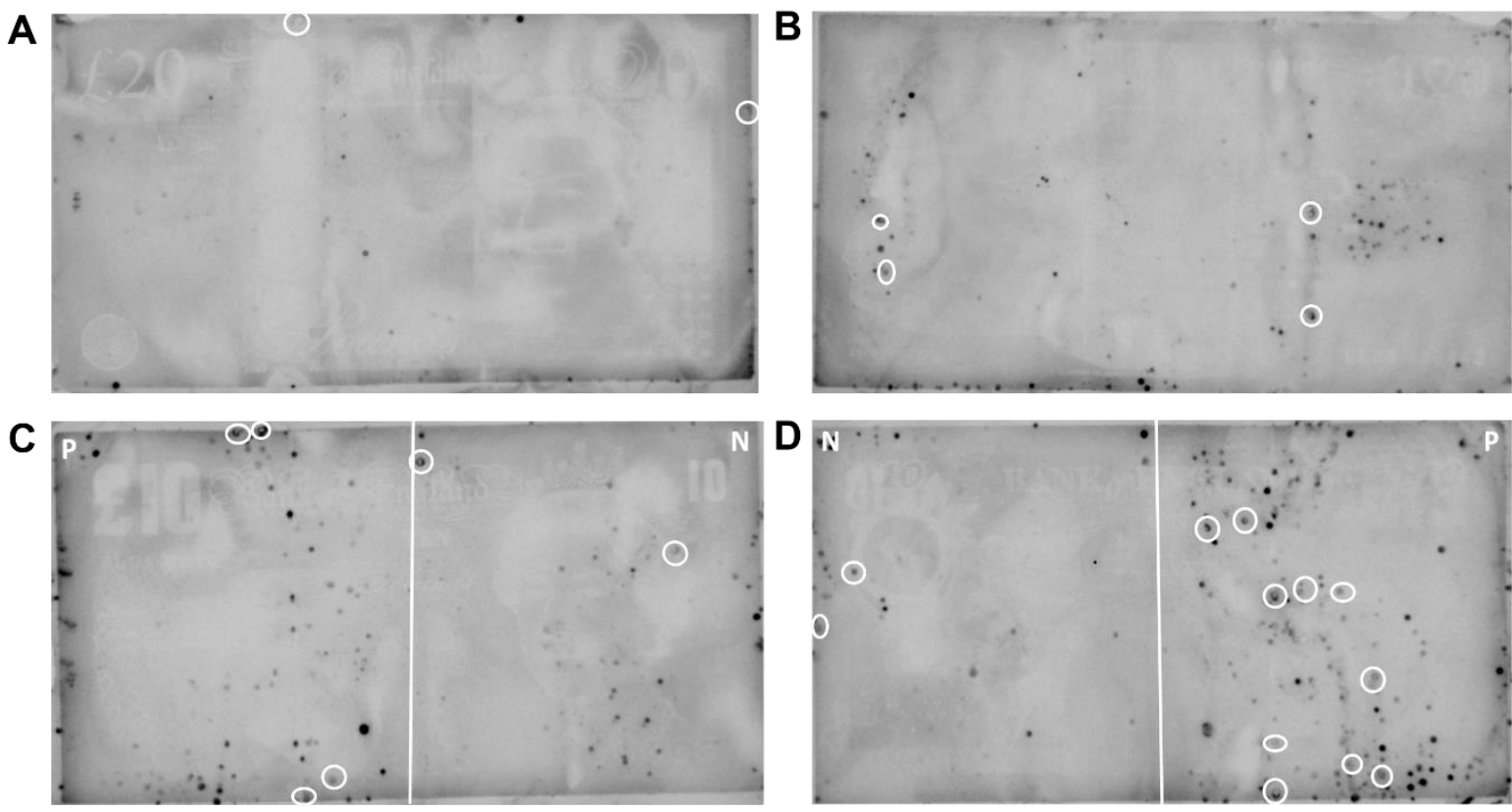

Figure 6. The immunostaining of one worn $£ 20$ banknote, and one worn $£ 10$ banknote obtained from general circulation within $7.5 \% \mathrm{w} / \mathrm{v}$ polyacrylamide gels. $(A)$ and $(B)$ show each side of the $£ 20$ banknote. (C) and (D) show each side of the $£ 10$ banknote, where half of the note is spiked with $10 \mathrm{mg}$ of street cocaine. The side of the note spiked with cocaine is labelled ' $P$ ', and the side without 'spiked' cocaine is labelled ' $N$ '. Chemiluminescent imaging was performed using a Fuji LAS-3000 imaging system, on high sensitivity mode for a total exposure time of $5 \mathrm{~min}$. The white circles indicate where air bubbles are present in the gels.

\section{Conclusions}

In this study, the development of a novel in-gel immunodetection technique for the qualitative detection of cocaine on the surface of banknotes has been reported. The results obtained here show that the immunodetection technique enables the direct immunostaining of the cocaine at the location at which it is present on the banknote. Overall, the results from the preliminary analysis of banknote samples performed during this study suggest that cocaine can be present at variable locations across the whole of the banknote. Based on the 
sporadic levels of contamination on the banknotes sampled, it is imperative that methods used by law enforcement agencies to determine cocaine contamination across the surface of notes provide a representative sample from the whole banknote.

\section{Acknowledgements}

The authors acknowledge the School of Chemistry, University of East Anglia for the studentship for S.v.d.H. The Home Office Centre for Applied Science and Technology (CAST) and Intelligent Fingerprinting Ltd. are gratefully acknowledged for their financial support. The authors are grateful to the Bank of England for providing the UK banknote samples and to the Norfolk Police Constabulary for providing the 'street' cocaine sample used during this study.

\section{References}

1 United Nations Office on Drugs and Crime, World drug report 2013, United Nations Publications, 2013.

2 L. V. Allen, M. L. Stiles, Clin. Toxicol., 1981, 18, 1043-1065.

3 T. Seddon, Brit. J. Criminol., 2006, 46, 680-703.

4 House of Commons Home Affairs Committee, The cocaine trade, The Stationary Office Ltd., London, 2010.

5 N. Dorn, J. Drug Issues, 2004, 34, 533-550.

6 D. A. Kidwell, S. A. Athanaselis, in: F. P. Smith (Ed.) Handbook of forensic drug analysis, Elsevier Academic Press, San Diego, 2005, pp. 235 - 275.

7 S. Jickells, A. Negrusz, Clarke's Analytical Forensic Toxicology, Pharmaceutical Press, London, 2008.

8 M. D. Hargreaves, K. Page, T. Munshi, R. Tomsett, G. Lynch, H. G. Edwards, J. Raman Spectrosc., 2008, 39, 873-880.

9 K. A. Ebejer, J. Winn, J. F. Carter, R. Sleeman, J. Parker, F. Körber, Forensic Sci. Int., 2007, 167, 94101.

10 R. Sleeman, F. Burton, J. Carter, D. Roberts, P. Hulmston, Anal. Chem., 2000, 72, 397A-403A.

11 K. Wimmer, S. Schneider, Forensic Sci. Int., 2011, 206, 172-177.

12 S. van der Heide, P. Garcia Calavia, S. Hardwick, S. Hudson, K. Wolff, D. A. Russell, Forensic Sci. Int., 2015, 250, 1-7.

13 K. A. Ebejer, G. R. Lloyd, R. G. Brereton, J. F. Carter, R. Sleeman, Forensic Sci. Int., 2007, 171, 165170.

14 F. E. Dussy, C. Berchtold, T. A. Briellmann, C. Lang, R. Steiger, M. Bovens, Forensic Sci. Int., 2008, 177, 105-111.

15 T. A. Briellmann, Dittmann, V., Dussy, F., Shwerzmann, T., Kriminalistik, 2001, 2, 4.

16 D. J. Dabbs, Diagnostic Immunohistochemistry, 2nd ed., Elsevier, Philadelphia, 2006.

17 A. H. Coons, H. J. Creech, R. N. Jones, Exp. Biol. Med., 1941, 47, 200-202.

18 R. R. Tubbs, K. Sheibani, S. D. Deodhar, W. A. Hawk, Cleveland Clin. Q., 1981, 48, 245-282.

19 S. Avrameas, Histochem. J., 1972, 4, 321-330. 
20 K. Murphy, P. Travers, M. Walport, Janeway's Immunobiology, 7th ed., Garland Science New York, 2008.

21 S. Desai, B. Dworecki, E. Cichon, Anal. Biochem., 2001, 297, 94-98.

22 K. Olden, K. M. Yamada, Anal. Biochem., 1977, 78, 483-490.

23 K. Burridge, Proc. Natl. Acad. Sci., 1976, 73, 4457-4461.

24 W. S. Adair, D. Jurivich, U. W. Goodenough, J. Cell Biol., 1978, 79, 281-285.

25 R. G. Anderson, E. Vasile, R. J. Mello, M. S. Brown, J. L. Goldstein, Cell, 1978, 15, 919-933.

26 R. T. Miller, R. Grantham, B. Lockett, C. Temple-Camp, J. Pang, Am. J. Foren. Med. Path., 2002, 23, 321-325.

27 G. Viel, A. Nalesso, G. Cecchetto, M. Montisci, S. D. Ferrara, Forensic Sci. Int., 2009, 193, 79-83.

28 M. Cingolani, M. Cippitelli, R. Froldi, V. Gambaro, G. Tassoni, J. Anal. Toxicol., 2004, 28, 16-19.

29 R. Leggett, E. E. Lee-Smith, S. M. Jickells, D. A. Russell, Angew Chemie Int. Ed., 2007, 46, 41004103.

30 S. van der Heide, D. A. Russell, J. Colloid and Interface Sci., 2016, 471, 127-135.

31 P. Hazarika, S. M. Jickells, K. Wolff, D. A. Russell, Angew. Chemie Int. Ed., 2008, 47, 10167-10170.

32 P. Hazarika, S. M. Jickells, D. A. Russell, Analyst, 2009, 134, 93-96.

33 P. Hazarika, S. M. Jickells, K. Wolff, D. A. Russell, Analytical Chemistry, 2010, 82, 9150-9154.

34 P. Hazarika, D. A. Russell, Angew. Chemie Int. Ed., 2012, 51, 3524-3531.

35 E. A. Ebejer, J. Winn, J. F. Carter, R. Sleeman, J. Parker, F. Körber, Forensic Sci. Int., 2007, 167, 94101.

36 J. M. Walker, in: J. M. Walker (Ed.) The Protein Protocols Handbook, 3rd ed., Springer, New York, 2009, pp. 177-185.

37 U. Laemmli, Nature, 1970, 227, 680-685.

38 P. Tijssen, E. Kurstak, Anal. Biochem., 1984, 136, 451-457.

39 G. B. Wisdom, in: J. M. Walker (Ed.) The Protein Protocols Handbook, Humana Press, New York, 2009.

40 G. R. Schonbaum, S. Lo, J. Biol. Chem., 1972, 247, 3353-3360.

41 S. van der Heide, D. A. Russell in E. Katz and J. Halámek (Eds.) Forensic Science: A Multidisciplinary Approach, Wiley-VCH Verlag GmbH, Weinheim, 2016, pp 283-297.

42 J. Moss, M. Sc. Thesis, University of Bristol, 1997. 